\title{
LA COLECCIÓN ARTÍSTICA DEL CANÓNIGO FRANCISCO RUIZ NOBLE Y LA SERIE DE LA VIDA DE JOSÉ DE ANTONIO DEL CASTILLO
}

\author{
ANA María Gómez Román ${ }^{1}$ \\ Universidad de Granada
}

\begin{abstract}
Francisco Ruiz Noble (Guadix, 1630-Granada, 1694) canónigo doctoral del templo metropolitano de Granada, fue un influyente religioso que cosechó el aprecio de otras tantas figuras dentro del ámbito cultural de la época. Hacedor de un gran bagaje intelectual y con refinados gustos en materia artística fue dueño de una importante colección de pintura en la que se encontraban representados artistas de la talla de Pedro Atanasio de Bocanegra, Sebastián Martínez, José de Cieza o Antonio del Castillo y su serie de la Vida de José (Museo Nacional del Prado).

Palabras clave: Coleccionismo; Francisco Ruiz Noble; Antonio del Castillo; Pedro Atanasio de Bocanegra; Sebastián Martínez; José de Cieza; José Risueño; Granada; Guadix.
\end{abstract}

\section{THE ARTISTIC COLLECTION OF THE CANON FRANCISCO RUIZ NOBLE AND THE SERIES "THE LIFE OF JOSEPH" BY ANTONIO DEL CASTILLO}

Francisco Ruiz Noble (1630 Guadix-1694 Granada), doctoral canon at the metropolitan temple of Granada, was an influential clergyman who earned the appreciation of other figures within the cultural sphere of the day. A person of great intellect and with a refined taste in artistic matters, he owned a significant painting collection in which major artists were represented, such as Pedro Atanasio de Bocanegra, Sebastián Martínez, José de Cieza or Antonio del Castillo and his series on The Life of Joseph (Museo Nacional del Prado).

Key words: Collecting; Francisco Ruiz Noble; Antonio del Castillo; Pedro Atanasio de Bocanegra; Sebastián Martínez; José de Cieza; José Risueño; Granada; Guadix.

Cómo citar este artículo / Citation: Gómez Román, Ana María (2017): "La colección artística del canónigo Francisco Ruiz Noble y la serie de la Vida de José de Antonio del Castillo”. En: Archivo Español de Arte, vol. 90, núm. 359, Madrid, pp. 229-242. doi: 10.3989/aearte.2017.15

A lo largo del siglo XVII los eclesiásticos fueron, junto con el estamento nobiliario y la monarquía, los grandes mecenas de las artes. A la par, durante esa centuria fueron adquiriendo una considerable presencia las galerías móviles de pintura de manera que las obras de arte se compraban, vendían, o heredaban, pero también se especulaba con ellas hasta el punto de surgir una abultada clientela con opinión propia en cuestiones artísticas ${ }^{2}$. En la ciudad de Granada fue fundamental el papel que desempeñaron algunos eclesiásticos quienes movidos por rasgos de generosidad y piedad se afanaron de manera muy especial bien en completar la ornamentación de determinados espacios religiosos o bien en el adorno de ámbitos de carácter privado, auspiciando así el trabajo de ciertos artistas hasta el punto de surgir, en algunos casos, una estrecha y

\footnotetext{
1 anaroman@ugr.es / ORCID iD: http://orcid.org/0000-0003-3363-1880.

2 Burke, 2001: 1383-1389.
} 
fluida relación entre cliente y artista. Fue así como un considerable número de pinturas y esculturas invadieron aquellos ámbitos señalados por estos promotores y clientes para su exhibición. A todo ello, un preciso grupo generó una tendencia acumulativa de obras de una cierta calidad artística, siendo quizá los arzobispos y canónigos quienes mostraron un mayor interés por esta práctica $^{3}$. Además, si analizamos en profundidad el prototipo de coleccionista que solía darse en esta ciudad observamos que, en su inmensa mayoría, eran individuos con un gusto encaminado al atesoramiento de obras de carácter religioso y devocional en detrimento de otros géneros, aunque hay que reconocer que también los hubo con inclinaciones más amplias y selectas. Este fue el caso de Francisco Ruiz Noble, un hombre de Iglesia cuya amplia personalidad le permitió alzarse como uno de los personajes más influyentes de la Granada del XVII y cuyo meteórico ascenso, dentro del estamento religioso, corrió paralelo a una cada vez mayor sensibilidad por las bellas artes. De hecho, fue un avezado coleccionista de pintura religiosa y muy especialmente de la obra del pintor cordobés Antonio del Castillo hasta el punto de atesorar una selecta representación de las obras más emblemáticas de este artista, nos referimos a la serie de la Vida de José conservada en el Museo Nacional del Prado.

\section{Aproximación biográfica}

Francisco Ruiz Noble nació en la localidad granadina de Guadix en septiembre de 1630. Fue bautizado en la iglesia parroquial del Sagrario el día 22 de ese mes, siendo su padrino su abuelo Alonso Vela de Roa, notario de profesión y regidor de la ciudad. Sus padres fueron Antonio Ruiz Noble, oriundo de Cascante (Navarra), y Magdalena Vela de Roa, y tuvo otros hermanos: Diego y Margarita Ruiz Noble. Dadas sus tempranas inclinaciones religiosas optó por abrazar la carrera eclesiástica en su ciudad natal de manera que para el año de 1641 ya había alcanzado el grado de presbítero ${ }^{4}$. Una vez instalado en la capital granadina realizó, entre los años 1648 y 1649, el correspondiente curso universitario de Instituta, prosiguiendo su formación académica en esta ciudad hasta el año de 1651 donde pasó a la Universidad de Sigüenza. Completada esta etapa ocupó diversos cargos antes de recalar en Granada, desde provisor en el obispado de Jaén, vicario y juez eclesiástico de Carzola, párroco de Castril, visitador de Huéscar, nombrado en este caso a instancias del cardenal de Toledo, hasta canónigo doctoral en la abadía de Baza en $1671^{5}$.

Sin embargo, fue en la ciudad de la Alhambra donde alcanzó los ministerios más influyentes de toda su dilatada carrera. Con todo, sus verdaderas miras estaban puestas en formar parte del gobierno del templo metropolitano, dado que el cabildo catedralicio gozaba de una rigurosa jerarquía que diferenciaba sustanciosamente sus retribuciones con las del resto de la comunidad religiosa. Esta circunstancia hacía aún más evidente el anhelo que gran parte del clero tenía por formar parte de este selecto grupo teniendo en cuenta que el arzobispado granadino, a diferencia de otras diócesis españolas, gozaba de unas abultadas rentas muy por encima de los 50.000 ducados anuales. Así fue ocupando diversas dignidades desde canciller de la universidad granadina durante los años 1678 y 1684, canónigo de la iglesia abacial del Sacromonte en 1680, provisor del arzobispado, y doctoral de la iglesia metropolitana a partir de 1684. Todo ello sin olvidar que en octubre de 1688 fue ascendido a archidiácono en sustitución de su anterior titular Eugenio Ribadeneira ${ }^{6}$. Más adelante, durante la grave enfermedad que mermó la capacidad de mando del arzobispo fray Alonso de los Ríos y Guzmán, fue designado gobernador arzobispal cargo que desempeñó hasta el nombramiento de Martín de Ascargorta en 1693 como nuevo metropolitano. También conviene apuntar que fue tal la diligencia de nuestro religioso que incluso tuvo una presencia activa en el proceso, incoado por el arzobispado en 1679, sobre la verificación de la

\footnotetext{
3 García Cueto, 2014: 363: 391. Gómez Román, 2014: 261-294.

4 Archivo Histórico Diocesano de Guadix (AHDG), órdenes 1640-1642. Caja 3541.

5 Jaramillo Cervilla, 2004: 272.

${ }^{6}$ Archivo Catedral de Granada (ACG), Actas 19, fol. 26.
} 
sobrenatural aparición de una estrella en el rostro de la efigie de la Virgen del Rosario venerada en la iglesia conventual de Santa Cruz la Real ${ }^{7}$.

Desde el preciso momento que Francisco Ruiz Noble se avecindó en Granada cosechó una merecida fama de hombre docto y entendido, por lo que no es de extrañar que se convirtiera en un referente dentro del contexto sociocultural de la época. En este sentido nos remitimos a su amistad con Pedro Suárez quien en 1672, recién llegado a ciudad del Darro procedente de Guadix y con el expreso deseo de proseguir su carrera eclesiástica, fue acogido y tutelado por nuestro religioso. Con el tiempo, Suárez se trasladó a Toledo convirtiéndose en capellán real y letrado de cámara del cardenal Luis Fernández Portocarrero y en esta ciudad escribiría su Historia de el obispado de Guadix y Baza $(1696)^{8}$. Pero Ruiz Noble cultivó, a su vez, el afecto de los artistas más representativos de la escuela granadina como el escultor Diego de Mora al que conoció cuando actuó en calidad de vicario general en la resolución de su expediente matrimonial con Ana de Soto9; o el pintor Pedro Atanasio de Bocanegra al que trató de forma directa allá por el año de 1679 durante el mencionado proceso de la Virgen del Rosario "llamé a pintores, y entre ellos à don Pedro Atanasio, Pintor de Su Magestad, que me dixo hiciese cerrar las puertas de la Iglesia porque la luz de la puerta ocasionaua con el reflexo dicha señal: despejose la Iglesia y auiendo cerrado la puerta no se vio dicha luz y Estrella" 10 . En este último caso, la relación entre ambos perduró más allá de dicho episodio hasta el punto que el pintor lo designó su albacea testamentario el 17 de enero de $1689^{11}$. Es más, gracias a esta confraternidad llegó a atesorar una quincena de trabajos del artista convirtiéndose, de este modo, en uno de sus mejores clientes. A pesar de ello no fue el único pintor por el que mostró inclinación ya que también admiró la obra de Felipe Gómez de Valencia quien, a su vez, le dedicó algunos de sus muchos dibujos y bocetos $^{12}$.

Por otra parte, nuestro prelado desempeñó un importante papel en la feliz consecución de diversas actuaciones que se emprendieron en el templo metropolitano durante las décadas finales del seiscientos. Primero en el año 1682 cuando recién incorporado al cabildo se hizo eco del expreso deseo del arzobispo fray Alonso Bernardo de los Ríos de cubrir las naves intermedias de la catedral, siendo uno de los pocos capitulares, junto con el prior Torres, que generosamente aportó el caudal necesario para dicho fin, en concreto unos 100 ducados ${ }^{13}$. Más adelante, el 29 de marzo de 1691, comisionado por los mismos beneficiarios, dado que en esos momentos era gobernador del arzobispado y hombre clave dentro de la curia granadina, se encargó junto con los doctores Bartolomé de Roa, Miguel de Rozas, Bartolomé Sánchez de Valera y Jerónimo de la Serna, de dictaminar sobre las condiciones de participación de Melchor de Aguirre en las obras de conclusión, según diseños de Alonso Cano, de la fachada del templo mayor ${ }^{14}$.

Por todo ello, y gracias a esta dilatada trayectoria, gozó de una gran solvencia económica muy por encima del resto de sus conciudadanos. Esta circunstancia, a la postre, le facilitó disponer del suficiente crédito como para invertir buena parte de su fortuna en la adquisición de obras de arte, constituyendo de esta forma una notable pinacoteca que quedó distribuida entre las estancias principales de su vivienda situada en la céntrica calle de San Jerónimo, junto casas principales de los Fonseca y próxima al colegio de la Compañía de Jesús. El inmueble en cuestión había sido comprado por el eclesiástico, por unos 2.000 ducados, a Magdalena Dávila de Fonseca y a su vástago Antonio Gómez de Montalvo, caballero del Hábito de Santiago además de generoso prócer de los jesuitas, y lindaba "por la parte de abajo con las casas del mayorazgo de los fon-

\footnotetext{
7 Ruiz Noble, 1680.

${ }^{8}$ Suárez, 1696: 314. Las primeras referencias historiográficas sobre Ruiz Noble nos las proporciona este erudito

9 Llevó a cabo el proceso matrimonial de Diego de Mora con Ana de Soto. Gallego Burín, 1988: 225 y ss.

10 Ruiz Noble, 1680: 1.

11 Gila Medina, 1997: 87-103.

12 Pérez Sánchez, 1986: 304-305.

13 Gila Medina, 2005: 191.

14 López López/Gila Medina/García Cueto, 2005: 1364.
} 
secas y por arriba con la botica que esta en dha calle junto a la pastelería"15. Previamente, y antes de instalarse definitivamente, se ocupó de realizar una completa remodelación con el fin de adaptarlo a sus exigencias entre las que se encontraban custodiar y distribuir su particular colección dentro del mismo. La obra, cuyo costo ascendió a unos 3.000 ducados, fue dirigida por los maestros alarifes Juan de la Torre y Jerónimo García. En esta misma morada fue donde expiró en la madrugada del día 5 de abril de $1694^{16}$.

\section{El testamento}

A pesar de haber realizado a lo largo de su vida varias mandas testamentarias, poco antes de morir y presintiendo el final de sus días, el canónigo dispuso sus últimas voluntades teniendo en cuenta que para la fecha el grueso de su fortuna, además de los bienes inmuebles y otros objetos de valor, se centraba en una amplia y selecta colección de pinturas. Estas últimas disposiciones fueron redactadas el 4 de abril de 1694 bajo la atenta mirada de los testigos José Omedes, Francisco de Arroyo, José de Santiago, Lorenzo de Rojas, Cristóbal de Torres, José Gil de Salazar y Juan Guerrero, este último paje del eclesiástico de unos 20 años ${ }^{17}$. En el citado documento de transmisión ordenaba que cada uno de los antedichos, y tras su deceso, debían ser retribuidos con algunas de sus joyas personales para "que sirba de memoria y de mi cariño y amistad". Pues bien, a las dos y media de la madrugada del día 5 de abril de 1694, y al poco de expirar, se procedió por parte de José Agustín de los Ríos y Bérriz, alcalde mayor de lo civil y teniente de corregidor de la ciudad de Granada, y José del Bario y Álvarez, escribano, al reconocimiento de los expresados refrendarios. Todos ellos afirmaron que eran plenos conocedores de estas disposiciones por lo que en presencia de los mismos se procedió a la pertinente apertura del testamento en el que figuraban como albaceas Manuel de la Fuente y Sandoval, capellán de la Capilla Real, Francisco Bonilla Noble de la orden de San Agustín Calzados y sobrino del eclesiástico, Juan Rico de Rueda, racionero del templo metropolitano de Granada, y Diego Ruiz Noble, hermano del finado. En el mismo, tal y como era preceptivo en la época, además de nombrar por heredera universal a su alma ordenaba decir mil misas por la misma. Igualmente disponía, en el caso de fallecer en su localidad natal, recibir cristiana sepultura en el panteón familiar emplazado en la iglesia conventual de San Francisco, mientras que si su deceso tenía lugar en Granada, como así ocurrió, debía ser en la "bobeda y entierro de los señores prebendados" del templo mayor de esta ciudad.

El cotejo de estas últimas voluntades nos proporciona algunos datos relevantes que completan en extensión su perfil biográfico y nos aclara dónde fueron a parar gran parte de sus bienes. Gracias a esta información documental sabemos que mantuvo un estrecho vínculo con la Congregación de San Felipe Neri, a cuyo santo fundador le tenía una especial devoción y aprecio. Los filipenses se habían establecido en la ciudad de la Alhambra en 1671, merced a las gestiones realizadas por una parte por el licenciado Dionisio del Barrio y por otra por el eclesiástico Francisco Hurtado de Mendoza, aunque no fue hasta la participación de Felipe Fermín, quien obtuvo la pertinente autorización mediante bula apostólica y facultad real para establecer dicha congregación en la ciudad de la Alhambra además de ser el instigador de la fundación del Oratorio en Baza, cuando dicha comunidad, integrada por sacerdotes y seglares sin necesidad de reglas y

15 Gracias al patronato instituido por la familia Fonseca se pudo construir el teatro del Colegio de San Pablo. Gila Medina, 2003: 433. En su origen el inmueble estaba vinculado a las propiedades del obispo accitano Juan de Fonseca. Magdalena como hija de Juan Dávila Fonseca, sobrino carnal del citado obispo, lo había heredado a su vez de su padre. Ruiz Noble adquirió la vivienda tras el sonado escándalo en que se vio envuelta la susodicha al pretender casarse con un caballero y que finalmente, ante la oposición de su propio hijo, le llevó a ingresar en un convento de clausura. Toral y Fernández de Peñaranda, 1995: 277-279.

${ }_{16}$ De la magnitud de su fortuna da cuenta el coste total de su funeral que ascendió a la nada despreciable suma de unos 6.153 reales.

17 Traslado del testamento que otorgó Francisco Ruiz Noble azerdiano que fue de la santta iglesia desta ciudad, Autos por Fallecimiento de Ruiz Noble (AHDG), 2 F (A) P-5. 
unidos por el vínculo de la caridad, realmente adquirió personalidad jurídica y administrativa. La estrecha amistad de Ruiz Noble con éste último, al que había conocido en tierras accitanas, supuso que tras su deceso recibiera un anillo, alba y casulla amén de un incensario, una naveta de plata y una capa de damasco. De igual modo, los filipenses se convirtieron en los grandes beneficiarios testamentarios del accitano aunque bien es cierto que existía una cláusula que disponía que en el caso de originarse algún problema con esta comunidad gran parte de sus propiedades deberían pasar a la Santa Escuela de Cristo, institución fundada en 1669 y con sede en el convento San Francisco Casa Grande, y de la que también era hermano. De ello deducimos que nuestro religioso, siguiendo los normas de esta última institución, además de practicar las reglas contemplativas propias de cualquier hombre de fe -caritativo, modesto, templado y sufrido- era, a su vez, fiel devoto de San Felipe Neri, santo protector de ambas instituciones. De hecho, por devoción al mismo ordenó en 1687 instituir en el templo metropolitano un aniversario, dotándolo de unos 188 reales y 8 maravedíes anuales, con su correspondiente procesión y misa, a concelebrar por el deán y cabildo respectivo, y para su soporte impuso un censo sobre su casa principal. Es más, uno de los principales motivos por los que decidió avecindarse en la calle de San Jerónimo fue su firme decisión de residir cerca de los filipenses. Incluso sopesó en un momento dado, y aconsejado por el padre Gregorio López, trasladar todas sus pertenencias -incluidas sus alhajas, ornamentos y la amplia colección de libros de Teología y Mística Moral- a la residencia de estos. Y aprestó que tras su óbito su vivienda quedara bajo la administración de los expresados religiosos estipulando en una cláusula de que si era arrendada los nuevos inquilinos debían firmar un compromiso expreso de facilitar el pago anual del citado aniversario catedralicio. $\mathrm{Y}$ así fue como la vivienda, una vez fallecido nuestro religioso, fue alquilada en tales condiciones al maestrante y caballero veinticuatro Sebastián Valero y Verdugo.

En otro orden, tras su deceso, y a pesar de la escrupulosidad con que se inventariaron sus bienes muebles, por equívoco se incorporaron al mencionado registro ciertos objetos que había disfrutado en usufructo y que sus legítimos dueños eran, en realidad, el presbítero Juan Alfonso García Navarro -en este caso propietario de varios muebles y siete pequeñas láminas con el tema de la Concepción - y Lucía Moreno -titular de una efigie de Nuestra Señora con vestido encarnado custodiada en el oratorio-. Una vez subsanado este error se procedió en primer lugar al ajuste de los gastos originados en torno a su sepelio y en segundo lugar a la liquidación de las cuentas pendientes del extinto, por lo que Pedro Zurbano percibió por este concepto unos 4.801 reales, el mercader Juan Luis Ponce otros 44 reales por la tasación de los libros y el maestro de albañilería Juan de la Torre unos 22,15 por el finiquito de una antigua deuda ${ }^{18}$.

\section{La colección de Ruiz Noble, la serie de la Vida de José de Antonio del Castillo y la tasación de pintura de José Risueño}

Sin duda alguna, y tal y como hemos expuesto, nuestro eclesiástico era un hombre de un gran bagaje intelectual y de una gran sensibilidad en lo que a materia artística se refiere, ya que no solo fue un ávido coleccionista sino amigo y benefactor de un buen número de pintores y escultores. No cabe la menor duda que tenía una especial inclinación hacia las obras de carácter religioso pero bien es cierto que, a diferencia de otros coetáneos, su interés por las bellas artes era mayor de lo habitual, en especial por el trabajo de artistas como Pedro de Mena, Pedro Atanasio de Bocanegra, Sebastián Martínez, José de Cieza o Antonio del Castillo.

Pues bien, para hacernos una idea aproximada de su colección conviene mencionar cuales eran las obras que atesoraba y cómo estaban distribuidas en el interior de su vivienda. Con respecto a las tres esculturas que aparecen contabilizadas en el inventario, y que había gozado en usufructo, eran propiedad de sus domésticas Lucía y María Moreno. Lucía era la legítima dueña

18 Por otra parte, el licenciado Juan José del Río realizó varios viajes a Alhama, a partir del 17 de enero de 1695, con el fin de inventariar las propiedades que Ruiz Noble tenía en esta población. 
de una hechura de San Antonio con el Niño "de mano de Mena" y una Virgen con el Niño, mientras que María Moreno de una talla pequeña de San José. Dichas imágenes habían estado custodiadas en el oratorio junto con varias láminas y "una tablita con dos puertas de Alberto Durero"19. Por lo que se refiere a los cuadros conviene puntualizar que estaban repartidos por todo el inmueble. De hecho, en el testero de la escalera principal colgaba un lienzo de Nuestra Señora de la Soledad, de vara y media de alto y sin marco. Por otra parte, y según el primer cotejo realizado por los albaceas el día 16 de abril, las obras contabilizadas, además de varias láminas que no se especifica ni el asunto ni el autor, eran las siguientes: un cuadro pequeño de San Felipe de medio cuerpo y marco dorado; dos cuadros de vara y media de Santa María Magdalena y de un Niño Jesús; un Niño Jesús Buen Pastor con marco dorado; once paisajes de poco más de una vara con marcos negros y dorados; dos "paises de florero con marco negro y dorado de a vara"; San Francisco de Sales y Santa Catalina ambos de "dos varas marco de nogal chapado"; San Pablo ermitaño de dos varas y media de largo y marco de nogal chapado; Nuestra Señora de Santa Ana de tres varas "poco más o menos, con marco negro"; un lienzo de la Encarnación "con marco negro alaminado de bara y media"; dos lienzos de San Antonio y San Jerónimo con "marcos negros y dorados de a vara"; Nuestra Señora de la Concepción de "tres baras moldura nogal chapada"; Cristo Crucificado del mismo tamaño y moldura; un Cristo de bronce con su peana de pino; "seis lienzos di gociete de la Historia de Joseph de poco mas de dos baras de ancho y una y media de alto todos con molduras doradas y el uno de una cabaña"; otro lienzo del Nacimiento de dos varas de alto con marco negro y dorado; la Adoración de los Reyes de dos varas y media de ancho con marco negro y dorado; El juicio de Salomón de dos varas de alto con marco negro y dorado; dos lienzos el uno de San Pedro y el otro de San Pablo ambos con marcos negros y dorados de una vara de alto cada uno y "un pais de dos baras y media de ancho y tres quartas de alto con marco negro y dorado". A estas piezas habría que sumarles las que se computaron en los aposentos del corredor alto: unos siete lienzos pequeños; y las de la sala principal, ubicada en el mismo corredor con vistas a la calle, donde colgaba un cuadro de San Pedro ad vincula de tres varas de alto y dos y media de alto sin marco; otro de Santa Bárbara de dos varas de alto con marco negro; un San Juan de Dios "de poco mas de bara de alto sin marco" y una cruz de madera con un Cristo pintado.

Tras este primer inventario los albaceas dispusieron otro más detallado y exhaustivo de acuerdo a la calidad de las piezas. En un primer momento, y de mutuo acuerdo, decidieron designar como tasador al pintor Juan de Sevilla el "qual dixo que rerespecto de sus muchas y continuados achaques no puede asistir ala tasacion de pintura" 20 . Tras la negativa de éste se vieron obligados a solicitar la ayuda de otro experto en la materia siendo José Risueño, quien a esas alturas reunía una más que sobrada maestría en el mundo del arte, finalmente el elegido. Resulta a todas luces que no era la primera vez que dicho artista actuaba como perito ni tampoco sería la última ${ }^{21}$. Gracias a su pormenorizado registro conocemos la autoría de gran parte de los cuadros de la pinacoteca de nuestro prelado siendo Antonio del Castillo (1616-1668) y Pedro Atanasio de Bocanegra (1638-1689) los pintores con más obra dentro de la colección. De hecho, Ruiz Noble guardaba con gran celo uno de los trabajos más culminantes del primero, la serie de la Vida de José, cuestión esta última fundamental dado que hasta la fecha sabíamos que uno de los pocos coleccionistas del pintor cordobés en Granada era Francisco de Torres Liñán, a la sazón contador de la catedral, tal y como nos detalla Palomino: "Y sobre todo en las Historiejas de mediano tamaño que superior, como lo manifestó en un juego de Cuadros de la Vida de Christo, y Martyrios de Apostoles, de los quales tenia algunos el Prior de la Vereda Don Pedro Garanza, en dicha Ciudad de Cordova; y otro juego de Historias de la Passion de Christo, que hizo para Don Francisco de Alfaro, de vara de alto; de los quales he visto quatro en la iglesia de San Phelipe

19 Tasaciones y almonedas delos bienes que quedaron por muerte de Don Francisco Ruiz Noble Arcediano que fue dela Sat Iglesia de esta ciudad (AHDG), pieza 1, fol. 10. Véase apéndice documental.

${ }_{20}$ Tasaciones y almonedas de los bienes que quedaron por muerte de Don Francisco Ruiz Noble Arcediano que fue dela Sat Iglesia de esta ciudad (AHDG), pieza 3.

${ }^{21}$ García Luque, 2013: 433-454. 
Fig. 1. Antonio del Castillo: José encuentra a sus hermanos en Dothán, ca.1655-1660. Madrid, Museo Nacional del Prado.

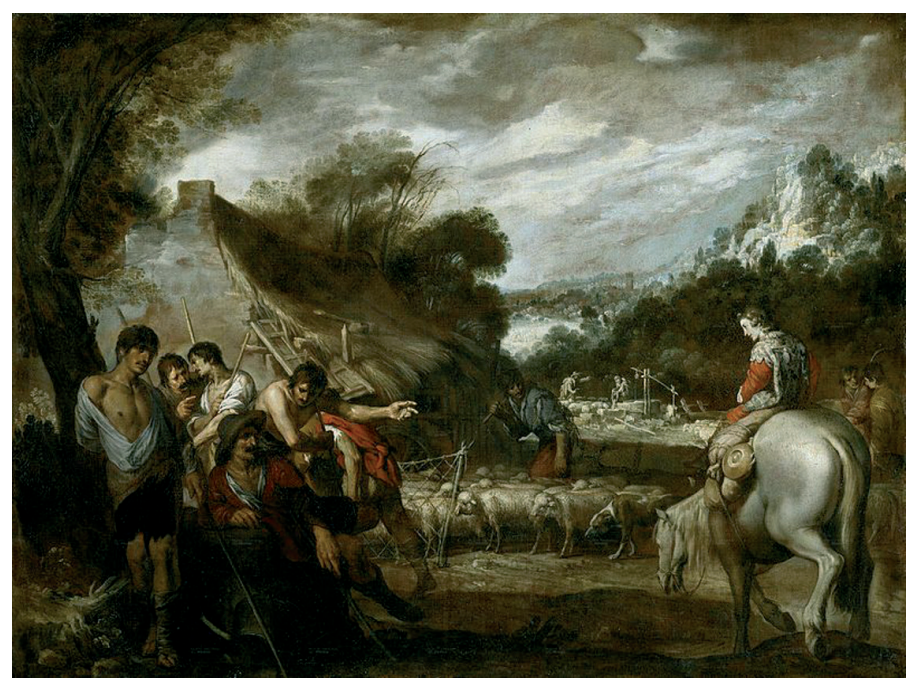

Neri de aquella Ciudad; y otros quatro en Granada en poder de Don Francisco de Torres y Liñan, contador y petriquero del Sacromonte, junto con otros quatro Paisillos del mismo Autor, de à tres cuartas de alto con Historiejas: la vna del Sacrificio de Abrahan; la otra del Hijo Prodigo; otra del triunfo de Judith; y la otra del Sueño de San Joseph en los zelos; todas hechas con singular gracia y primor" 22 .

En realidad, debemos advertir que hallar obras del pintor cordobés en la ciudad de la Alhambra no era muy habitual dado que el mercado estaba abastecido, en su gran mayoría, con obras de artistas de la escuela granadina como Bocanegra o Juan de Sevilla. Por ello los seis lienzos a los que nos referimos, y que hoy día forman parte de los fondos del Museo Nacional del Prado: José encuentra a sus hermanos en Dothán (fig. 1); José vendido por sus hermanos (fig. 2); La castidad de José; José explica los sueños al faraón; El triunfo de José en Egipto (fig. 3) y José ordena la prisión de Simeón, reflejan muy bien el tipo de coleccionista que era ${ }^{23}$. Por lo que respecta a la información que aparece sobre dichos cuadros en los documentos consultados constatamos que en el primer registro realizado nada más fallecer nuestro prelado se especificaba al respecto lo siguiente: "Seis lienzos di gociete de la Historia de Joseph de poco mas de dos baras de ancho y una y media de alto todos con molduras doradas y el uno de una cabaña". La citada serie del hijo de Jacob, una historia bíblica basada en la esperanza y la fe, fue pintada por Antonio del Castillo a mediados de siglo aunque desconocemos en origen para quién. Tampoco se puede pasar por alto que el pintor bien pudo tener en cuenta algunos escritos de la época como los del agustino José Laínez. Por una parte la obra dedicada al conde duque de Olivares, El privado christiano deducido de la vida de Ioseph y Daniel que fueron valanza de los validos en el fiel contraste del pueblo de Dios (Madrid, 1641); y por otra El Joséph Virrey de Egipto (Madrid, 1652). En cualquier caso lo sustancial del asunto es el apego que el pintor demuestra hacia el paisaje, trabajado bajo la influencia de Abraham Bloemaert, donde combina los asuntos religiosos con escenarios rústicos ${ }^{24}$. En opinión de Mindy Nancarrow representa la "crítica velada a las autoridades cordobesas por no proporcionar pan, lo que provocó los disturbios de 1652 " 25 y su

22 Palomino de Castro, 1724: 365.

23 En el inventario del Museo Nacional del Prado figuran con el siguiente número de registro: P00951, P00952, P00953, P00954, P00955, P00956. En: <https://www.museodelprado.es/coleccion/obras-de-arte?cidoc:p14_carried out_by@@@m:author@@ecidoc:p131_E82_p102_has_title=Castillo\%20Saavedra,\%20Antonio\%20del> (consultado 12-12-2015). Presentan similares medidas: $109 \times 143 \mathrm{~cm}$.

${ }^{24}$ Navarrete Prieto, 1998: 172-174.

25 Nancarrow, 1990: 116-128. 

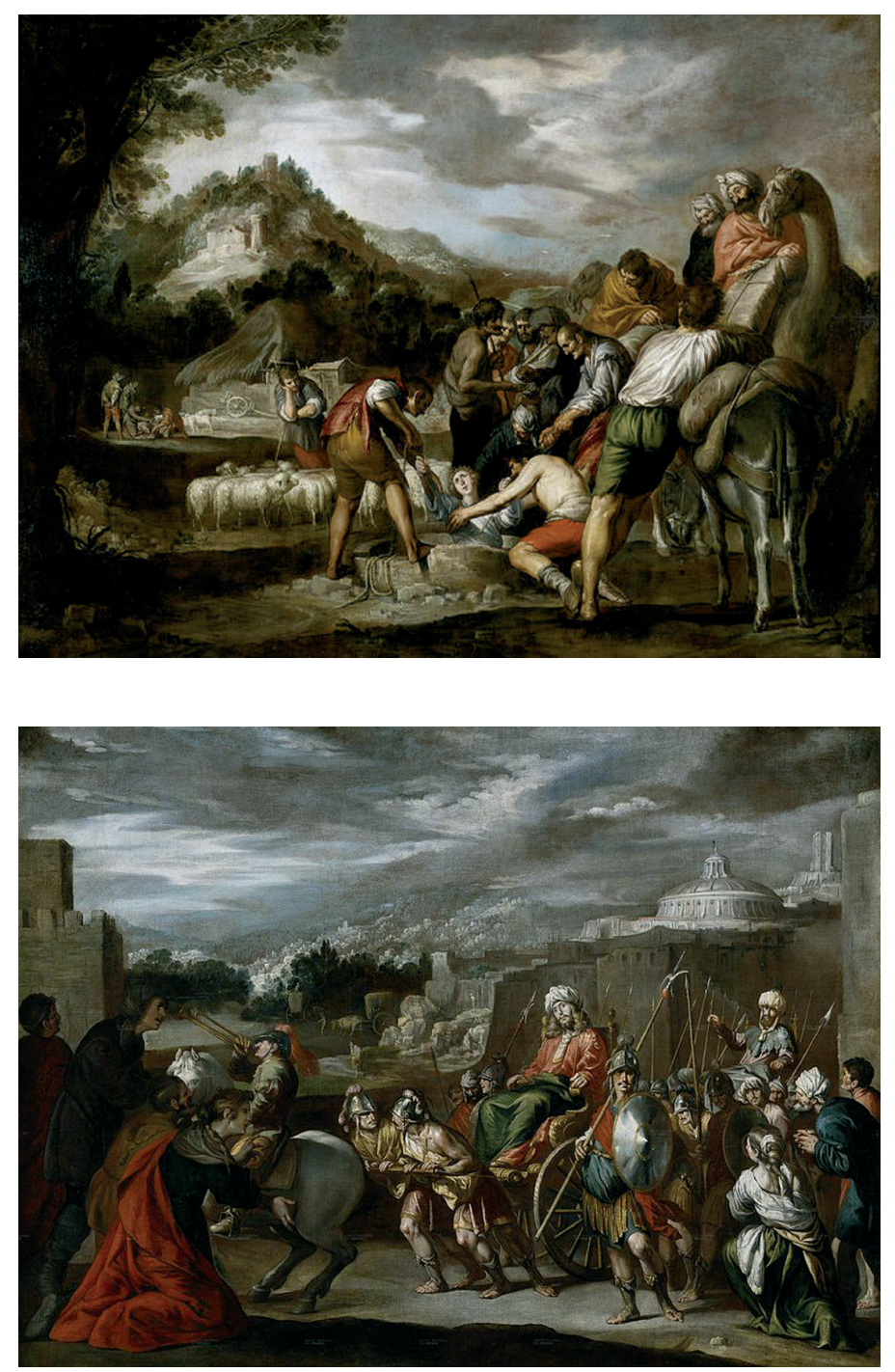

Fig. 2. Antonio del Castillo: José vendido por sus hermanos, ca. 1655-1660. Madrid, Museo Nacional del Prado.

Fig. 3. Antonio del Castillo: El triunfo de José en Egipto, ca.1655-1660. Madrid, Museo Nacional del Prado.

comitente bien pudo ser José de Valdecañas y Herrera ${ }^{26}$. Aventurándonos en el campo de la hipótesis podríamos suponer, dado que Valdecañas era granadino, que pudo existir algún tipo de concomitancia entre el caballero Veinticuatro de la ciudad de Córdoba y el accitano hasta el punto que, una vez fallecido el primero, nuestro coleccionista bien pudo hacerse con este ciclo, y con algún lienzo más del pintor cordobés, a través de sus herederos ${ }^{27}$.

Es muy probable que el cuadro registrado por Risueño como Los antecedentes de la Predicación de San Juan ("dos baras y quartta de ancho y media uara de alto") podría ser el que perteneció en su día a la colección López Cepero y que en la actualidad forma parte del Museo

26 Esta hipótesis ha sido planteada por Nancarrow/Navarrete Prieto, 2004: 187.

27 Información genealógica de José Valdecañas y Herrera. Archivo Histórico Nacional (AHN), Consejo de Inquisición, 14778, expediente 10. José de Valdecañas y Herrera, natural de Granada y avecindado en Córdoba dado que en 1623 se convirtió en oficial de la Santo Oficio de la Inquisición y ejerció como abogado de dicha institución. Fue el comitente del lienzo el Arcángel San Rafael con destino a la casa consistorial pintado en 1652 firmado en la parte inferior derecha: "Votis, et sollicitudines/D,D, Josephi d Valde/cañas, et Herrera/Senatoris. Antonius/ d Castillo P A 1652" (Ayuntamiento de Córdoba). Nancarrow/Navarrete Prieto, 2004: 340-342. 
Cerralbo donde en un primer momento se catalogó como "La Cabaña" hasta que una reciente restauración ha puesto de manifiesto la existencia, en su borde izquierdo, de la figura recortada de San Juan. Está inventariado como El encuentro de San Juan Bautista con unos campesinos a la salida del desierto $(106 \times 136 \mathrm{~cm})$, datado entre 1655 y 1660 y es una obra firmada ${ }^{28}$. En cualquier caso desconocemos el paradero del resto de los cuadros que Risueño también adjudica al pintor cordobés: La Historia de Salomón (de "dos baras de alto y siette quinta de altto"), El Nacimiento (con las mismas medidas que el anterior), la pareja de lienzos de San Pedro y San Pablo (ambos de "uara de alto y tres quarttas de ancho") y la tabla de San Francisco de Sales.

Con todo, el pintor con mayor presencia en la pinacoteca del accitano era Pedro Atanasio de Bocanegra. De este artista el canónigo atesoraba una Inmaculada Concepción, un Cristo, un San Onofre de "dos baras de ancho y bara y tercia de alto", la pareja de lienzos de Jessé y María de "tres cuartas de alto y media vara de ancho"; un Niño Pastor de "vara y quarta de alto y tres quarttas de ancho"; San Antonio y San Jerónimo de "vara de alto y tres cuartas de ancho"; unos cinco lienzos con las mismas medidas (una vara y media) que representaban a San Francisco Javier, San Miguel, Santa Catalina, Santa Teresa y San Francisco de Paula; dos lienzos de San Juan y La Magdalena de "uara y tercia de ancho y tres cuartas de alto" y finalmente un San Felipe Neri con unas medidas de "tres cuartas de alto y dos tercias de ancho". La colección se completaba con dos floreros del desconocido pintor Bernardo Xirón; un cuadro de la Encarnación de José de Cieza (1656-1692); otro de San Pedro Ad Víncula copia de Esteban de Rueda (ca. 1609-1687) así como un cuadro original de este último, en concreto una Inmaculada, y La Virgen con el Niño y Santa Ana de "siette cuartas de ancho y bara y quartta de altto" del pintor jiennense Sebastián Martínez (ca. 1615-1667). Cerraba la lista unos once paisajes y un lienzo de un perro, todos ellos anónimos.

Por otra parte Juan de Cervantes, "maestro de platero y contraste", se encargó de tasar todas las piezas de orfebrería. De su relación se advierte que en el oratorio se almacenaban diversos cucharones, platos, vasos, un cáliz sobredorado y una patena además de vinajeras de hojalata, un ostiario, campanilla y piletas. Según su tasación estos objetos alcanzaban un valor de $6.518,8$ reales. Por lo que se refiere al mobiliario y enseres domésticos el peritaje fue realizado por Juan Rodríguez Márquez. En su lista aparecen anotadas una Santa Teresa de Jesús con su cerco de flores ( 3 reales), una cruz de Caravaca con figuras de bronce (6 reales), un relicario de plata escarchada (4 reales), una hechura de San Antonio de talla de tres cuartas de alto con peana ordinaria ( 88 reales), un altar portátil ( 24 reales), un frontal de damasco ( 77 reales), un misal (180 reales) y dos cajones repletos de ornamentos que incluían casullas, cíngulos, etc.

\section{Una almoneda singular}

Con frecuencia la liquidación de los bienes testamentarios era un acto de gran trascendencia dentro del ámbito comercial puesto que permitía hacer frente tanto a las deudas pendientes del difunto como a los previsibles gastos generados por su sepelio o a las obras pías, aniversarios, etc., instituidos por el mismo. Esto suponía que los entendidos en arte estaban al tanto de esta operación económica que, en el caso de darse las condiciones oportunas, les permitiría hacerse con obras de artistas de primera fila que en otras circunstancias les habría sido prácticamente imposible adquirir dado el alto valor que solían alcanzar en el mercado. Influía, obviamente, el tipo de colección que se ofertaba ya que solía ser fiel reflejo de la sensibilidad artística de su anterior titular. Con todo, cuando se trataba de personajes de un cierto bagaje intelectual las colecciones artísticas eran conocidas de antemano por amigos y afines, puesto que, tal y como era costumbre en la España del XVII, sus propietarios no habían tenido reparo en exhibirlas en $v i_{a}^{29}$. Por tanto aquellos que gozaban del suficiente capital, o que tenían ciertas pretensiones

\footnotetext{
28 Nancarrow/Navarrete Prieto, 2004: 325-326.
}

29 Morán Turina/Portús Pérez, 1997. 
sociales, veían en estas operaciones una oportunidad única para hacerse con obras que, en la mayoría de las ocasiones y como hemos expuesto, codiciaban y sabían de antemano de su mérito. En el caso que nos ocupa la almoneda testamentaria se desarrolló entre dos escenarios. El primero fue la propia residencia del religioso y el segundo la céntrica plaza de Bib Rambla. En ambos casos fueron los lotes de pintura los primeros que salieron a la venta y los que también despertaron un mayor interés. Así, y ante una enorme expectación, la almoneda dio comienzo el 21 de abril de 1694 en sus casas principales bajo la supervisión de un fiscal nombrado ex profeso y de los respectivos albaceas. José de Mena, hijo del afamado escultor Pedro de Mena y religioso de profesión, fue uno de los primeros en acudir a la cita haciéndose de una colgadura de "rasso de la china" valorada en 310 reales. Por su parte Antonio de Lamas adquirió el cuadro del Perro (60 reales); mientras que la que había sido antigua doméstica del eclesiástico, Lucía Moreno, el lienzo de Santa Bárbara (33 reales). Petronila Gómez pujó por el lienzo de la Concepción de pintura ordinaria por unos 11 reales y Melchor de Cisneros por el cuadro de la Concepción con moldura chapada y dorada grande por unos 400 reales. Al día siguiente, tras reanudarse de nuevo la licitación, Francisco de Ortega compró las tres láminas de Jesús, La Virgen y El Niño por 610 reales, Simón de León la lamina de la Virgen por unos 33 reales; Alonso Monzón el cuadro de San Felipe Neri por 60; Luis Muñoz el grande de Santo Cristo por 400 reales y finalmente Fabián de Robles las siete laminas de papel pequeñas por 28 reales.

Empero, las obras de mayor mérito fueron adjudicadas en un mismo día siendo sus licitadores dos hombres vinculados a la iglesia, avezados en cuestiones estéticas, y expertos conocedores de las tendencias artísticas del momento. Nos referimos a Simón Pérez de Costela y a Miguel Muñoz de Ahumada. En el caso del primero conviene advertir que tenía una personalidad bien definida dado que su formación en el colegio de San Dionisio Areopagita, y más tarde el desempeño del beneficio de la parroquia de Santa María la Alhambra, le había forjado un singular gusto artístico. Pues bien, Costela adquirió en el transcurso de la mañana del 23 de abril los lienzos pintados por Bocanegra de San Francisco, Santa Teresa, San Francisco Javier, San Miguel, Santa Catalina y San Francisco Javier, desembolsando por ello un total de 900 reales $^{30}$. Por lo que se refiere a Miguel Muñoz de Ahumada se hizo a lo largo de la tarde de un total de 8 cuadros: los seis de la Vida de José y La Cabaña de Antonio del Castillo y el lienzo de la Virgen, Santa Ana y el Niño pintado por Sebastián Martínez ${ }^{31}$. Pagó por ellos la nada despreciable suma de 2.500 reales. Al día siguiente, Carlos Fernández de Lillo, escribano de cámara de la Real Chancillería de Granada, pujó por los once paisajes por unos 100 reales.

La licitación cambió de escenario el día 26 trasladándose a la plaza de Bib Rambla. Como era de esperar al ser un escenario público el remate congregó a un gran número de curiosos y entendidos entre los que se encontraban Antonio Vélez, quien se hizo de un cuadro por unos 92 reales; Juan de Córdoba, de los cuadros apaisados de San Antonio y San Jerónimo por 200 reales; y Juan de Pineda por un lado de los dos lienzos de San Juan y La Magdalena por unos 150 reales y por otro de los dos cuadros de flores por 70 reales. Por su parte, José de Torres se agenció del lienzo de la Encarnación por unos 38 reales; Lorenzo de Morales del biombo (152 reales) y Miguel Fernández de Almarcho del cuadro de San Onofre desembolsando, en este caso, unos 110 reales. Un día después, y en el mismo escenario, Andrés Díaz de Heredia se hizo con la cruz de madera con un Cristo pintado por unos 4 reales; Ana Pardo del lienzo de La Tentación (20 reales); Ignacio Antonio de Cuenca del lienzo grande de San Pedro (180 reales) y Juan García Malo de Molina de los dos escritorios chapados en carey por unos 600 reales. Curiosamente para julio todavía quedaban por vender varios lienzos, lo que dio pie a que el caballero Malo de Molina el día 10 de dicho mes se agenciase del cuadro grande "del pueblo de Israel passando el mar bermexo".

Los sucesivos meses se reservaron para el remate de las joyas, la orfebrería y la espléndida biblioteca del eclesiástico. De esta manera, el 27 de mayo Pablo Diamante adquirió buena parte

\footnotetext{
${ }^{30}$ Tasaciones y almonedas delos bienes que quedaron por muerte de Don Francisco Ruiz Noble Arcediano que fue dela Sat Iglesia de esta ciudad (AHDG), pieza 3, fol. 28.

31 Tasaciones y almonedas delos bienes que quedaron por muerte de Don Francisco Ruiz Noble Arcediano que fue dela Sat Iglesia de esta ciudad (AHDG), pieza 3, fol. 29.
} 
de las alhajas, a excepción de las de plata, que habían formado parte del oratorio por unos 2.200 reales. El juego compuesto por un cáliz, copa y vinajeras así como un ostiario y todos los utensilios de plata fueron a parar a Lucía Moreno por un importe total de 1.447,17 reales. La librería se puso a la venta a partir del 30 de junio y fue rematada en varios días. A las pertinentes citaciones acudieron bibliógrafos como Bernardo Salinas; Manuel de la Fuente; Tomás Rodríguez, vecino de Málaga; Juan de Frías, fiscal del Arzobispado; Mateo de la Peña, presbítero; Juan Luis de León, mercader de libros, o el letrado Tomás de Castilla Peregrín.

\section{Corolario: la Vida de José en la colección de Miguel Muñoz de Ahumada}

De todo lo anteriormente expuesto colegimos que uno de los lotes con mayor estimación fue la serie de la Vida de José pintado por Antonio del Castillo. Miguel Muñoz de Ahumada, provisor, canónigo y tesorero de la catedral, se hizo con el mismo puesto que conocía de antemano la amplia estima que la obra del Castillo tenía entre los entendidos de la época. Además, con la adquisición de estos seis cuadros, junto con el lienzo de La Cabaña también del mismo pintor, demostraba su particular gusto por la pintura de paisaje, algo que era poco habitual entre los coleccionistas granadinos. Pero, al igual que Ruiz Noble, este singular religioso disponía de un gran discernimiento en cuestiones artísticas ya que había tenido una formación y una trayectoria muy parecida al accitano. Cualidades a las que podíamos sumar su pleno juicio sobre el trabajo de los principales artistas que trabajaban en la ciudad dada su condición de visitador de las iglesias y conventos granadinos. Conviene recordar que este notable eclesiástico había nacido en la localidad granadina de Montefrío y que realizó una completa formación académica, primero como colegial del Colegio Imperial de San Miguel y más adelante como estudiante de Cánones en la Universidad donde se graduó brillantemente el 12 de abril de $1638^{32}$. Su ascenso social quedó consolidado en 1656 al obtener una canonjía en el templo metropolitano de Granada lo que le permitió en 1667 convertirse en flamante tesorero ${ }^{33}$. Todo ello vendría a justificar que tuviera muy clara la inversión que hacía al adquirir los lienzos del artista cordobés aunque tras su óbito serían nuevamente enajenados, junto con el resto de sus bienes muebles, en pública almoneda ${ }^{34}$.

Por otra parte, en relación a la citada serie, en el siglo XIX aparece documentada como propiedad del aristócrata Pedro Victoria Ahumada y Diéguez, caballero maestrante y Veinticuatro de Granada, miembro de la granadina Hermandad de la Caridad y del Refugio y Gentilhombre de Cámara de S.M ${ }^{35}$. Tras desprenderse de la misma fue finalmente adquirida por el Gobierno, a instancias del Marqués de la Vega de Armijo, mediante R.O. 8 de abril de 1863 con destino al Museo de la Trinidad ${ }^{36}$. En el catálogo de dicho museo, firmado por Cruzada Villaamil, se atri-

\footnotetext{
32 Pruebas de Miguel Muñoz de Ahumada, Archivo Universidad de Granada (AUG), legajo, 1031.01 y Certificación pruebas de curso 1638, (AUG), legajo 1584.053. Por lo demás, en el año 1643 fue autorizado por la Real Chancillería de Granada para ejercer como abogado, el 15 de enero del año siguiente tomó posesión de la canonjía doctoral de Baza, y en 1649 completaba su meteórica carrera al ser designado fiscal de Visita del Tribunal de la Santa Inquisición en la diócesis de Guadix. Más adelante fue provisor y vicario examinador general de la abadía de Baza, vicario general del arzobispado de Sevilla y rector del granadino Colegio de San Miguel en los años de 1659 y 1678.

${ }_{33}$ Méritos Miguel Muñoz de Ahumada 1670, Archivo General de Indias (AGI), Indiferente, legajo 200, 46.

${ }_{34}$ Muñoz de Ahumada realizó testamento en 1695. Desconocemos quien adquirió la serie de Antonio del Castillo tras la almoneda.

35 Pedro Pablo de Victoria Ahumada y Diéguez Duárez de la Cuadra nació en Pataz (Perú) en 1805 aunque su familia era de ascendencia granadina con propiedades en la localidad de Motril, entre ellas la conocida Casa de la Palma. Tras vivir en Granada, ciudad en la que estudió en su universidad, en 1850 se convirtió en Director del Sindicato de Riegos de Lorca. Fue gobernador de Toledo y Oficial de Clase de Terceros en el Ministerio de Fomento. En 1873 se hizo cargo de forma interina de la Dirección General de Instrucción Pública. Falleció en Motril en 1890. La pertenencia de la serie de la Vida de José a Victoria Ahumada ya fue señalada por Mindy Nancarrow y Benito Navarrete (Nacarrow/Navarrete Prieto, 2004: 187).

36 Álvarez Lopera, 2004: 74.
} 
buyó a Pedro de Moya: "En los seis cuadros que de su mano atesora este Museo se ven grandes y excelentes condiciones de colorista, notable correccion de dibujo, brio en la entonación y buen gusto artístico" 37 . Resulta altamente interesante este hecho porque su adquisición por parte del Ministerio de Fomento, y por la cual se desembolsó una considerable suma, fue precisamente por su atribución al pintor granadino dado que por aquellas fechas eran muy pocas las obras conocidas de este artista. Incluso la prensa señaló al respecto lo siguiente: "para aumentar mas y mas el valor de estos cuadros añadiremos que hemos creido ver en uno de ellos el retrato de Pedro de Moya"38. Finalmente, tras la desaparición del Museo de la Trinidad, los lienzos se incorporaron a los fondos del Museo Nacional del Prado donde se custodian en la actualidad ${ }^{39}$.

\section{APÉNDICE DOCUMENTAL}

“-Los siete lienços que son la historia de Joseph y la cavaña todos de mano de Antonio del Castillo con marcos dorados los que tassa a quarenta ducados cada uno que ymportan $3 \& 080$.

- Otro lienço de dos baras y media de ancho y dos de alto con marco negro y dorado, en ochocientos rreales de vellon $\& 800$.

- Otro lienço de dos baras de alto y siette quinta de altto. Con marco negro y dorado dela historia de Salomon de mano del dho Ant. ${ }^{\circ}$ del Castillo en treinta ducados \& 330.

-Otro del mismo autor y tamaño y marco que el antecedente del nacim. ${ }^{\text {to }}$ en trecientos y treinta $\mathrm{r}^{\mathrm{s}}$ $\& 330$.

- Dos cuadros el uno de San Pedro y el otro de San Pablo ambos de uara de alto y tres quarttas de ancho con las molduras negra y doradas a cien $\mathrm{r}^{\mathrm{s}}$ cada uno \& 200

-Otro lienço de dos baras y quartta de ancho y media uara de alto del mismo Antonio del Castillo de quien son los antecedentes de la predicación de San Juan Baptista en cientto y cincuenta rreales \& 150.

- Otro de siette cuartas de ancho y bara y quartta de altto con marco negro y dorado de nra Sa el niño $\mathrm{y}^{\text {ra }} \mathrm{S}^{\text {ra }}$ Santta Ana de mano de Seuastian martines el de Jaen en quatro cientos $\mathrm{r}^{\mathrm{s}} \& 400$.

\section{De Atanasio}

- Otro de dos baras y media de altto y dos de ancho con marcho chapado de nogal dorado de $\mathrm{n}^{\text {ra }} \mathrm{S}^{\mathrm{a}}$ dela concepción de mano de Don Pedro Atanasio en seiscientos $\mathrm{r}^{\mathrm{s}} \& 600$.

- Otro del mesmo tamaño y marco y autor del San ${ }^{\text {to }}$ Cristo en cincuenta ducados \& 550.

-Otro de dos baras de ancho y bara y tercia de alto con moldura chapada y dorada del mismo autor de san onofre en duscientos $r^{\mathrm{s}} \& 200$.

-Dos cabeças una de Jesé y otra de maria de tres cuartas de alto y media uara de ancho con molduras chapadas y caprichos dorados del mismo autor en trecientos $\mathrm{r}^{\mathrm{r}} \& 300$.

-Octro lienço del mismo autor de uara y quartta de alto y tres quarttas de ancho con marco tallado y dorado del niño pastor en trescientos y cinqta rreales $\& 350$.

- Dos quadros el uno de $\mathrm{S}^{\mathrm{n}}$ San Antonio y el otro de san G. ${ }^{\text {mo }}$ ambos del mismo autor con marcos negros y dorados de una bara de altto y tres quarttas de acnho ambos en ducienttos y quareta $\mathrm{r}^{\mathrm{s}} \& 240$.

- Otros dos lienços del mismo autor de uara y media en quadauno el uno de san Franco Javier y el otro del angel San Mig ${ }^{1}$ con marcos chapados y dorados en ducientos y cincuenta $\mathrm{r}^{\mathrm{s}}$ cada uno \&500.

- Otro del mismo autor de San Francisco de Sales de uara y tercia en quadrado con marco chapado y dorado en ducientos y quranta $\mathrm{r}^{\mathrm{s}} \& 240$.

-Otro del mismo autor de santa Catalina del tamaño y molduras que el antecedentte en ducientos y quaranta reales $\& 240$.

- Otros dos lienços del mismo autor de uara y media encuadrado cada uno y ambos con moluduras chapadas y doradas el uno de santa theressa y el dho de san Fraco de Paula a ducientos y cuarenta reales cada uno $\& 480$

37 Cruzada Villaamil, 1865: 137. Los cuadros se compraron por 38.500 reales.

38 "Variedades de la Capital. Me gustan". En: El Clamor Publico, Periódico del Partido Liberal, Madrid, 18-IV1863: 3-4.

39 Estuvieron atribuidos a Pedro de Moya hasta 1913. 
- Dos lienços el uno de san Juan y el otro de la magna deuara y tercia de ancho y tres quarttas de altto con molduras talladas y doradas ambos de mano del dho Don Pedro Atanasio en trecientos $\mathrm{r}^{\mathrm{s}}$ los dos $\& 300$.

- Otro del rretrato de san Phelipe Neri con moldura dorada de tres cuartas de alto y dos tercias de ancho en sesentta $r^{\text {s }} \& 060$.

-Dos floreros de mano de don Bernardo Xiron de tres cuartas de alto con marcos negros y dorado en cien $\mathrm{r}^{\mathrm{s}}$ cada uno $\& 200$.

-Once paises de bara de ancho y dos tercias de altto con marquillos dorados y negros a doce $\mathrm{r}^{\mathrm{s}}$ cada uno \& 132.

-Una laminica de $n .{ }^{\text {ra }}$ s. ${ }^{\text {a }}$ con marco de pino negro de una tercia de altto en quince $r^{\mathrm{s}} \& 015$.

-Un lienço de bara y tercia de ancho y uara de alto con marco negro de la encarnación de mano de don Joseph de Ciezar en sesentta y seeis reales \&066

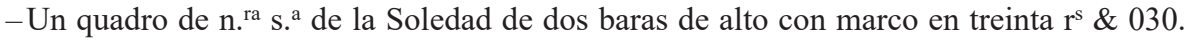

- Otro en que esta pintado un perro de una bara en quadrado en cincuenta reales \&050.

- Siette estampas de papel de una quartta en cuadrado con marcos pintados negro y blancos a tres $\mathrm{r}^{\mathrm{s}}$ cada una \&021.

- Un lienço de dos baras y media de ancho y bara y quartta de altto sin marco de Jesucristo en el desiertto en sesenta reales $\& 060$.

-Un biombo de seis paños de dos baras y quarta de altto y bara de ancho cada paño en trecientos $r^{\mathrm{s}}$. $\& 300$.

- Otro quadro de santa baruara con marco negro de siette cuartas de alto y bara y quarta de altto en sesenta reales \&060.

- Otro de san pedro aduincula de tres baras de ancho y dos y media de altto en duscientos y cincuenta $\mathrm{r}^{\mathrm{s}}$ el qual es copia de Esteuan de rueda $\& 250$.

\section{Oratorio}

- Un lieço de $n^{\text {ra }} \mathrm{S}^{\text {ra }}$ de la Concepción de dos baras de alto y bara y media dancho con moldura dorada de mano de esteuan de rueda. En trecientos y cinquenta $\mathrm{r}^{\mathrm{s}} \& 350$.

- Una tablita con dos puertas de Aluerto durero de media tercia de altto en cientto y veinte $\mathrm{r}^{\mathrm{s}}$. \& 120 .

- Una laminica de una caueça de nuestra sra de una tercia de altto con la moldura de euano pintada en cobre en noventa $\mathrm{r}^{\mathrm{s}}$. \&090.

- Una tabla de san Francisco de Sales con una moldura negra de mas de tercia de altto de manto de Atanasio en sesenta $r^{\mathrm{s}}$. \&060.

- Una lamina en cobre do dos tercia de ancho y media bara de altto con molduras de euano a cientto y cinqueta $\mathrm{r}^{\mathrm{s}}$ cada una $\& 300$.

- Dos laminas pintadas en cobre de media uara de ancho y una terçia de altto la una de santta Cecilia y la dhia de la cena de Baltasar con molduras de euano en treçienttos y cincuenta las dos \&350.

- Otra lamina de dos trecias de altto y una de ancho pintada en cobre con moldura de ebano de Jesuchistro quando echo los Judios del templo en ciento y cincuenta \&150.

- Una laminica de San Carlos Borromeo de una quartta de alto y media tercia de ancho con moldura de euano con moldura de cobre en quince $r^{s} \& 015$.

- Una laminica obalada pintada en cobre con moldura de euano fileteada de san Fran. ${ }^{\text {co }}$ de mano de don pedro Atanasio en treinta reales \&030.

- Otra laminica de Susana en el uaño de una quarta de ancho y media tercia de altto con moldura de pinto en quince $r^{\mathrm{s}} \& 015$.

- Otra laminica del tamano dela antecedentte dela fuente del Sacramento con bidriera en quince $\mathrm{r}^{\mathrm{s}}$ dicha lamina de $\mathrm{n}^{\mathrm{ra}} \mathrm{s}^{\mathrm{ra}}$ de la concepçion pintada en lienzo con vidriera de media bara de altto y tercia de ancho con marco de peral en treinta $\mathrm{r}^{\mathrm{s}}$. \&030.

- Quatro estampas las dos con marco de flores de seda y la una de san Joseph con marco de peral y la otra con marco de papel y seda en ueinte $r^{\mathrm{s}} \& 020$.

- Un pedaço de rrasso en questa bordada $n^{\text {ra }} \mathrm{s}^{\text {ra }}$ con el niño en seis $\mathrm{r}^{\mathrm{s}}$. \&006.

- Una cruz en questa pintada un santto cristo de mano de don Joseph de Ciezar en cinquenta $\mathrm{r}^{\mathrm{s}}$. \&050.

- Una cruz en questa pintado un satto cristo en quatro $r^{s} \& 004$.

- Dos laminas con marco de pinto con sus bidrieras bordadas de seda la una de san Fran ${ }^{\mathrm{co}}$ y la dicha de san Pedro en treinta las dos \&030.

$12 \& 169 \mathrm{RS}$ 
y en la forma referrida hiço $\mathrm{s}^{\text {ta }}$ tasación la qual jura a dia y auna cruz en forma de derecho auerla hecho uien y fielmente ajuste la saber y entender sin graude nii agrauio contra ninguna delas partes senguo en conocimiento que de la Pintura tiene y tiempo presente y lo firmo

Joseph Risueño de laconcha (sic)

Ante Pedro Fernández de orttega".

\section{BIBLIOGRAFÍA}

Álvarez Lopera, José (2004): El Museo de la Trinidad en el Prado. Madrid: Museo del Prado.

Burke, Marcus (2001): "Los mercados del arte en la España del siglo XVII”. En: Bernardo Ares, José Manuel (coord.) El Hispanismo anglonorteamericano. Aportaciones, problemas y perspectivas sobre Historia, Arte y Literatura españolas (siglos XVI-XVIII). Córdoba: CajaSur, pp.1383-1389.

Cruzada Villaamil, Gregorio (1865): Catálogo provisional historial y razonado del Museo Nacional de Pinturas. Madrid: Manuel Galiano.

Gallego Burín, Antonio (1988): José de Mora. Granada: Universidad.

García Cueto, David (2014): "La pintura italiana en la Granada del Barroco: artistas y coleccionistas, originales y copias". En: Cruz Cabrera, José Policarpo (coord.) Arte y cultura en la Granada renacentista y barroca: relaciones e influencias. Granada: Universidad, pp. 363-391.

García Luque, Manuel (2013): "José Risueño, un artista versátil al servicio de la Catedral de Granada". En: Laboratorio de Arte, 25, Sevilla, pp. 433-454.

Gila Medina, Lázaro (1997): "Nuevos datos para la vida y obra del pintor real Pedro Atanasio de Bocanegra -Testamento, codicilio, inventario y tasación de su patrimonio artístico-". En: Cuadernos de Arte de la Universidad de Granada, 28, Granada, pp. 87-103.

Gila Medina, Lázaro (2003): "Contribución al estudio del antiguo Colegio de San Pablo de los Jesuitas -hoy Facultad de Derecho- de Granada”. En: Cortés Peña, Antonio Luis/López-Guadalupe, Miguel Luis (2003): Estudios sobre Iglesia y Sociedad en Andalucía en la Edad Moderna. Granada: Universidad, p. 433.

Gila Medina, Lázaro (2005): "La última etapa constructiva: de 1650 a 1704”. En: Gila Medina, Lázaro (coord.) El Libro de la Catedral de Granada. Granada: Cabildo Metropolitano de la Catedral de Granada, vol. 1, p. 191.

Gómez Román, Ana María (2014): "Patronazgo artístico y coleccionismo eclesiástico en la Granada barroca". En: Cruz Cabrera, José Policarpo (coord.) Arte y cultura en la Granada renacentista y barroca: la construcción de una imagen clasicista. Granada: Universidad, pp. 261-264.

Jaramillo Cervilla, Manuel (2004): “Aproximación histórica a la vida y obra de Pedro Suárez”. En: Boletín del Instituto de Estudios Pedro Suárez, 17, Guadix, p. 272.

López López, Pedro/Gila Medina, Lázaro/García Cueto, David (2005): “Corpus documental”. En: Gila Medina, Lázaro (coord.) El Libro de la Catedral de Granada. Granada: Cabildo Metropolitano de la Catedral de Granada, vol. 2, p. 1364.

Morán Turina, Miguel/Portús Pérez, Javier (1997): El Arte de mirar: la pintura y su público en la España de Velázquez. Madrid: Itsmo.

Nancarrow, Mindy (1990): “Narrative Meaning in Antonio del Castillo's The Life of Joseph”. En: Gazette des BeauxArts, 156, Paris, pp. 116-128.

Nancarrow, Mindy/Navarrete Prieto, Benito (2004): Antonio del Castillo. Madrid: Fundación de Apoyo a la Historia del Arte Hispánico.

Navarrete Prieto, Benito (1998): La pintura andaluza del siglo XVII y sus fuentes grabadas. Madrid: Fundación de Apoyo a la Historia del Arte Hispánico, pp. 172-174, figs. 301-306.

Palomino de Castro y Velasco, Antonio (1724): El Parnaso Español Pintoresco Laureado. Tomo Tercero. Con las vidas de los Pintores, y Estauarios Eminentes Españoles, que con sus heroycas obras han ilustrado la Nacion: y de aquellos estrangeros ilustres, que han concurrido en estas Provincias, y las han enriquecido con sus eminentes Obras; Graduados según la serie de el tiempo, en que cada uno floreció: para eternizar la memoria, que tan justamente se vincularon en la posteridad tan sublimes, y remontados espiritus. Madrid: Juan García Infançon.

Pérez Sánchez, Alfonso (1986): Historia del dibujo en España. Madrid: Cátedra, pp. 304-305.

Ruiz Noble, Francisco (1680): Discurso sobre la calificacion de la luz en forma de estrella que se vio entre dos cejas de la Imagen de N.S. del Rosario el dia 26 de Iunio de 1679. Granada: Raimundo de Velasco.

Suárez, Pedro (1696): Historia de el Obispado de Guadix y Baza. Madrid: Imprenta Antonio Román.

Toral y Fernández de Peñaranda, Enrique (1995): "De otros tiempos”. En: Boletín del Instituto de Estudios Gienneses, 155, Jaén, pp. 277-279.

Fecha de recepción: 03-II-2016

Fecha de aceptación: 19-V-2016 